\title{
Recherche en psychothérapie : La pointe de l'iceberg et le reste
}

Peter Schulthess

À loccasion du Congrès PSY à Berne (cf. compte-rendu du colloque dans ce cahier), Jens Gaab a fait un discours remarquable sur la recherche en psychothérapie. Jens Gaab est professeur de psychologie clinique et de psychothérapie à l'université de Bâle. Ses explications sont restituées ci-après sous forme de résumé.

Dans le recherche en psychothérapie, une quantité énorme de données ont été rassemblées depuis ses débuts. En 1909, il existait tout juste deux études, il y en a aujourd'hui selon pubmed. gov (une sorte de Google de la recherche médico-clinique) entre 8.000 et 9.000 par an, donc un comportement des chercheurs plus qu'actif. L'hypothèse selon laquelle il existe pour les troubles psychiques des causes spécifiques et des traitements spécifiques est un moteur de cet activisme de recherche. Cette hypothèse revient à Freud, qui voulait intégrer ainsi la psychanalyse (psychothérapie) dans le système de la médecine.

Dans la mesure où jusquà aujourd'hui toutes les approches thérapeutiques doivent démontrer, pour être reconnues scientifiquement, quelles ont la même efficacité que d'autres processus (ou qu'elles sont même plus efficaces, quelles agissent plus spécifiquement), la recherche y contribue sous la forme d'une concurrence entre ceux qui seraient meilleurs et plus efficaces.

Étrangement, les premières hypothèses sur la psychothérapie reviendraient à l'hypothèse d'un magnétisme animal de Franz Anton Mesmer. Nous connaissons aujourd'hui environ 200 différents procédés psychothérapeutiques et 500 méthodes. La Society of Clinical Psychology (une division de l'APA) classe les procédés et méthodes selon les points de vue de la médecine basée sur des preuves et a élaboré des critères qui s'appliquent à la scientificité.

Sur la base de la recherche, les premiers doutes sont apparus sur l'hypothèse que les troubles psychiques ont des causes spécifiques et pourraient être soignés par des traitements spécifiques. Il fut supposé que les différents procédés thérapeutiques montraient des succès quelque peu similaires (verdict du dodo). Cette hypothèse publiée par Rosenzweig en 1936 fut la base à la formulation de facteurs généraux, respectivement communs, d'efficience des différents concepts thérapeutiques. Comment pourrait s'expliquer le fait que des concepts thérapeutiques avec des théories différentes, voire opposées, donnaient lieu à des succès thérapeutiques similaires ? Cétait un premier indice qu'il devait bien exister dans la psychothérapie d'autres facteurs d'efficiences que des interventions spécifiques pour des troubles spécifiques. On a conclu que les méthodes seraient identiques ou interchangeables et que c'est la croyance du thérapeute dans l'efficacité de ses interventions spécifiques qui permet la réussite. L'intervenant a utilisé l'image d'un iceberg pour démontrer qu'il ne suffit pas que la recherche analyse simplement la pointe qui émerge de l'eau et néglige les 91,68 pour cent du volume qui repose sous l'eau. Mais qu'il y a-t-il en dessous de la surface de l'eau pour ce qui est des interventions? Le placébo!

Il est possible que le placébo ait été d'abord introduit par l'Église catholique pour diagnostiquer la possession par le diable. On projetait de l'eau bénite sur une personne à expertiser. Si elle criait et se déchaînait, cétait la preuve de la possession et de la présence du diable, ce qui signifiait l'indication d'un processus d'exorcisme. De l'eau normale était alors aussi projetée à titre de comparaison. Si la personne criait aussi et se démenait, cétait alors la preuve que le diable nétait pas en elle, mais qu’elle était folle. Elle était internée. C'est ainsi que le placébo a sauvé quelques vies (Kaptchuk et al., 2009).

Dans des essais avec le magnétisme de Mesmer sous Louis XVI, il a été démontré que l'effet de
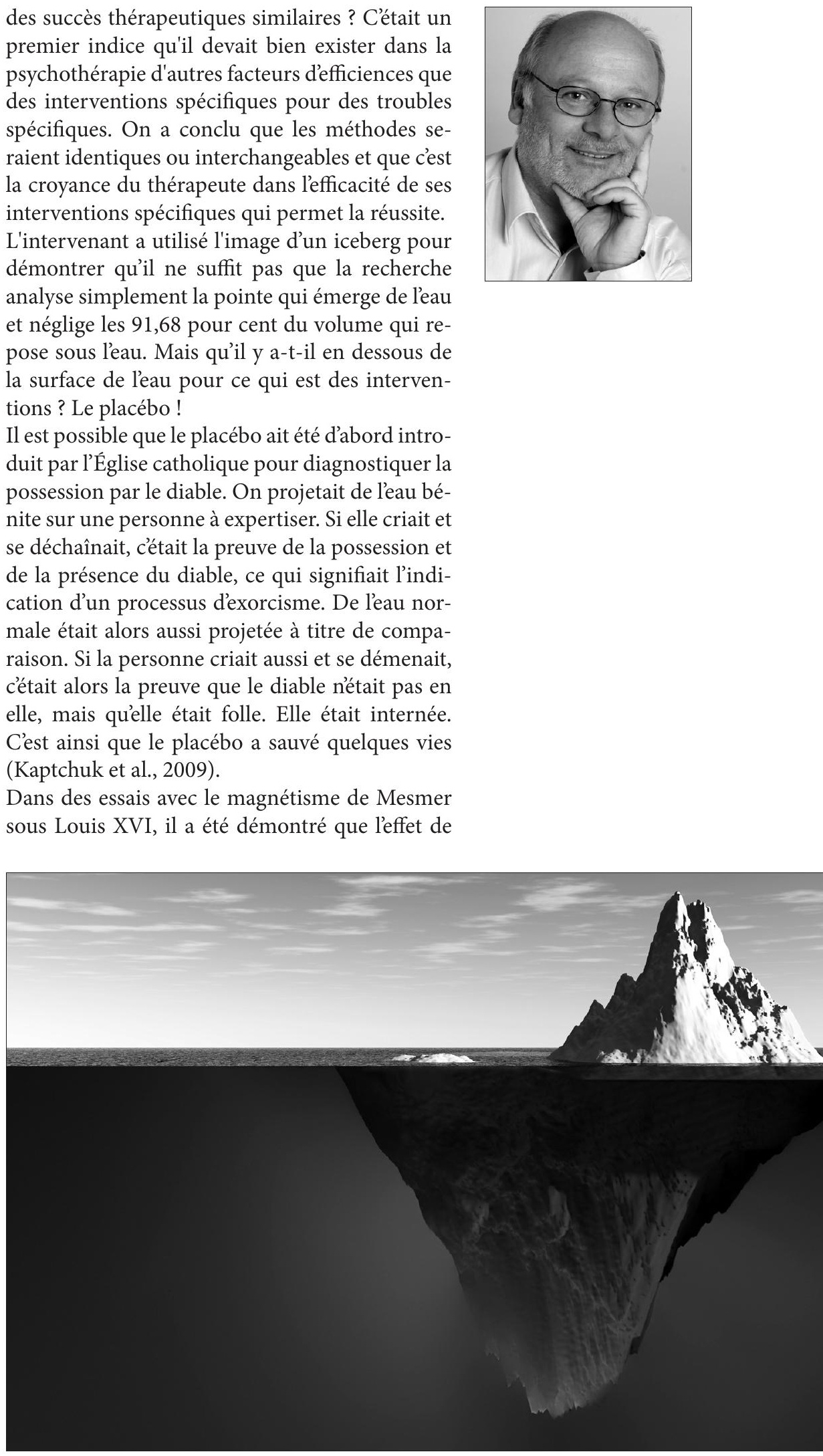
guérison souhaité apparaissait alors aussi même lorsque personne ne se tenait derrière le rideau, mais que le sujet de test le croyait simplement parce qu'on le lui avait dit (Franklin et al., 2008 [1784]).

Des études sur le placébo pendant des opérations (l'opération nétait pas du tout effectuée, mais on coupait simplement superficiellement et on recousait) ont démontré que le placébo était efficace dans 65 pour cent des cas. Dans le traitement de patients souffrants, même 80 pour cent. La recherche a démontré une tendance en hausse de l'augmentation de l'efficacité du placébo, selon la méthode avec laquelle il est administré (Jonas et al., 2015). Des essais avec des antidépresseurs montrent que le placébo est efficace dans 75 pour cent des cas (Khan \& Brown, 2015).

Des essais ont également été faits dans le domaine non médical : On a remis des violons Stradivarius et des violons classiques du marché à des violonistes professionnels. Lorsque le violoniste ne savait pas qu'il jouait sur un Stradivarius, il évaluait l'instrument comme étant plus mauvais que ceux du marché (Fritz et al., 2012). Des essais avec le vin ont démontré que des sujets de test appréciaient mieux le même vin lorsquon leur disait qu'il était très cher que lorsqu'on leur disait qu'il s'agissait d'un vin bon marché (Plassmann et al., 2008).

Une expérience avec le placébo a également été faite avec les femmes de ménage. Elles ont été réparties en deux groupes. L'un devait simplement accomplir sa tâche de nettoyage. On a dit à l'autre que le ménage était du sport et très sain. Les deux groupes ont fait le même travail. Des différences bluffantes sont apparues : Les « sportives » ont perdu du poids, leur IMC a diminué, la pression sanguine et la teneur en graisse dans les tissus ont baissé.

Dans une autre étude, il a été démontré que la prise régulière d'un placébo abaissait la mortalité sur le long terme. Le risque était de moitié moindre que chez ceux qui ne prenaient pas régulièrement le placébo (Crum \& Langer, 2007). L'attente semble donc influencer l'effet. Mais, pas seulement : Ce sont aussi les relations au thérapeute (expérimentateur) et à la « substance active » qui influencent l'effet. Par la suite, le questionnement fut modifié : C'est manifestement le « donner du sens » qui est le facteur d'efficience, car le placébo en lui-même n'a aucun effet (Moermann \& Jonas, 2002).
Quel est le lien avec la psychothérapie ? Avec des médicaments, on peut comparativement effectuer simplement des études contrôlées du placébo. Dans la psychothérapie, cela est plus difficile. Dans des études contrôlées, dans lesquelles un groupe a été traité par une psychothérapie spécifique et un groupe de contrôle a reçu seulement une " thérapie " non directive, de support et empathique, il a été démontré que, pour une équivalence structurelle des groupes comparatifs sur le plan de la quantité, du format et de la durée des séances thérapeutiques, ainsi que de la formation du thérapeute et des restrictions du contenu, des résultats similaires ont été obtenus. Des différences sont apparues uniquement lorsque les deux groupes nétaient pas structurellement identiques (Baskin et al., 2003).

Une autre étude arrive à la conclusion que, dans l'efficacité de la psychothérapie, seulement 1 pour cent est attribué à des facteurs spécifiques, 34 pour cent à des facteurs hors de la thérapie et 65 pour cent à des facteurs non spécifiques (Cuijpers et al., 2012).

Ici, on doit déjà être fortement convaincu pour continuer à adhérer à la thèse selon laquelle des troubles psychiques auraient une cause spécifique et pourraient être traités par une méthode thérapeutique spécifique. Des études plus récentes démontrent aussi que le verdict du dodo continue à s'appliquer (Leichsenring et al., 2014) . La psychothérapie est-elle un placébo ? L'intervenant a tiré une conclusion intermédiaire :

- des effets spécifiques de la psychothérapie sont alors possibles lorsquon organise en conséquence les conditions de contrôle

- dans une comparaison juste, la différence nest pas significative

- les psychothérapies diffèrent seulement sur le plan de la théorie, mais pas dans leur effet

- des placébos avec un effet psychothérapeutique sont possibles et efficaces (avec des effets modérés à importants)

- Surpris?

Ci-dessous, Gaab est revenu sur Rosenzweig qui fut le premier à publier sur des facteurs communs implicites des différents concepts thérapeutiques et a alors poursuivi avec Jerome Frank (1986) qui décrivait la psychothérapie comme une transformation de la signification. Il faisait référence au «grand débat psychothérapeutique » et renvoyait à lédition allemande qui vient de paraitre du 
livre Die Psychotherapie Debatte. Was Psychotherapie wirksam macht (Wampold et al., 2018). On y trouve une vue d'ensemble de la recherche en psychothérapie et une représentation du modèle contextuel de Wampold, qui arrive à la conclusion que des facteurs complémentaires, comme les caractéristiques du thérapeute, jouent un rôle important dans leefficacité de la psychothérapie. Les auteurs démontrent que la psychothérapie marche. Des facteurs defficience cliniquement significatifs sont : des objectifs communs du patient et du thérapeute, l'empathie, une bonne relation, lestime. Au milieu du classement : personnalité du thérapeute, authenticité, adaptation, attente. Négligeables : Différences des approches thérapeutiques, compétence, méthodes, adhérence (Wampold \& Imel, 2015). Ce sont des facteurs d'efficience déjà décrits par Carl Rogers (Roger, 2007 [1957]).

Dans une recherche personnelle, pas encore publiée concernant la recherche sur les placébos dans la psychothérapie, il a pu démontrer que le fait que le placébo soit administré d'une manière dissimulée ou ouvertement ne joue aucun rôle. Avec une administration ouverte et des explications correspondantes du thérapeute, l'effet était même plus important qu'avec une administration dissimulée. C'est le signe que la relation est un « verum » (Gaab et al., 2018).

Dans une perspective théorique, la psychothérapie se situe à une proximité inconfortable des effets du placébo. Comment s'en libérer pour pouvoir sétablir comme une science sérieuse? Selon l'intervenant, il ne suffit pas de se fonder sur lévidence empirique, lexpertise clinique et les attentes des patients, cela nécessite en plus davantage de principes éthiques qui reposent sur : Autonomie, bienfaisance, non-maléficience et équité. Il a renvoyé ici à une nouvelle publication sur l'éthique de la psychothérapie (Trachsel et al., 2018).

Parmi les principes éthiques, on trouve l'explication ouverte aux patients sur ce que nous, en tant que thérapeutes, savons et sur ce que nous faisons, mais également sur ce que nous ne savons pas. Ce qui est déterminant pour la réussite thérapeutique c'est une relation ouverte, digne de confiance et de partenariat.

Dans la discussion, on sest interrogé si la recherche ne devait pas simplement s'appliquer encore plus à mieux comprendre les facteurs spécifiques d’efficacité. Gaab a répondu avec la question « pourquoi voudrait-on continuer alors à regarder autant la pointe de l'iceberg ? » La future recherche en psychothérapie ferait mieux de se concentrer sur les 92,68 pour cent de l'iceberg qui repose sous le visible.

\section{Bibliographie}

Baskin, T.W., Tierney, S.C., Minami, T. \& Wampold, B.E. (2003). Establishing specificity in psychotherapy: a meta-analysis of structural equivalence of placebo controls. Journal of Consulting and Clinical Psychology, 71(6), 973-979.

Crum, A. J. \& Langer, E. J. (2007). Mind-set matters. Exercise and the placebo effect. Psychological Science, 18(2), 165-171.

Cuijpers, P., Driessen, E., Hollon, S. D., van Oppen, P., Barth, J. \& Andersson, G. (2012). The efficacy of non-directive supportive therapy for adult depression: a meta-analysis. Clinical psychology review, 32(4), 280-291.

Frank, J.D. (1986). Psychotherapy - the transformation of meanings: discussion paper. Journal of the Royal Society of Medicine, 79(6), 341-346.

Franklin, B., Majault, M. J., Le Roy, J. B., Sallin, C. L., Bailly J.-S., d'Arcet, J., de Bory, G., Guillotin J.-I. \& Lavoisier, A. (2008 [1784]). Report of the commissioners charged by the king with the examination of animal magnetism. International Journal of Clinical and Experimental Hypnosis, 50(4), 332-363.

Fritz, C., Curtin, J., Poitevineau, J., Morrel-Samuels, P. \& Tao, F.C. (2012). Players preferences among new and old violins. Proceedings of the $\mathrm{Na}$ tional Academy of Sciences, 109(3), 760-763.

Gaab, J., Locher, C. \& Blease, C. (2018). Placebo and Psychotherapy: Differences, Similarities and Implications. International review of neurobiology, 138, 241-255.

Jonas, W. B., Crawford, C., Colloca, L., Kapchuk, T. J., Moseley, B., Miller F. G., Kriston, L., Linde, K. \& Meissner, K. (2015). To what extent are surgery and invasive procedures effective beyond a placebo response? A systematic review with meta-analysis of randomized, sham controlled trials. BMJ open, 5(12), e009655. https://doi.org/10.1136/bmjopen2015-009655

Khan, A. \& Brown, W.A. (2015). Antidepressants versus placebo in major depression: an overview. World Psychiatry, 14(3), 294-300.

Kaptchuk, T. J., Kerr C.E. \& Zanger, A. (2009). Placebo controls, exorcism and the devil. The Lancet, 374(9697), 1234-1245. 
Leichsenring, F., Salzer, S., Beutel, M.E., Herpertz, S., Hiller, W., Hoyer, J. \& Huesing, J. (2014). Longterm outcome of psychodynamic therapy and cognitive-behavioral therapy in social anxiety disorder. American Journal of Psychiatry, 171(10), 1074-1082.

Moerman, D.E. \& Jonas, W. B. (2002). Deconstructing the placebo effect and finding the meaning response. Annals of Internal medicine, 136(6), 471-476.

Plassmann, H., O’Doherty, J., Shiv, B. \& Rangel, A. (2008). Marketing actions can modulate neural representations of experienced pleasantness. Proceedings of the National Academy of Sciences, 105(3), 1050-1054.

Rogers, C.R. (2007 [1957|). The necessary and sufficient conditions of therapeutic personality change.
Psychotherapy: Theory, Research, Practice, Training, 44(3), 240-248.

Rosenzweig, S. (1936). Some implicit common factors in diverse methods of psychotherapy. American Journal of Orthopsychiatry, 6(3), 412-415.

Trachsel, M., Gaab, J. \& Biller-Andorno, N. (2018). Ethische Standards in der Psychotherapie. Göttingen: Hogrefe.

Wampold, B. E. \& Imel, Z.E. (2015). The great psychotherapy debate: The evidence for what makes psychotherapy work. New York: Routledge.

Wampold, B.E., Imel, Z. E. \& Flückiger, C. (2018). Die Psychotherapiedebatte. Was Psychotherapie wirksam macht. Göttingen: Hogrefe.

\section{Peter Schulthess est membre du conseil} d'administration de l'ASP.

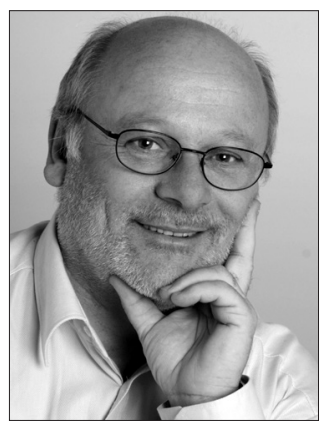

Comme cela est abordé dans le rapport du Congrès PSY 2018, l'allocution de Dirk Helbing sur le thème "Utilisation de l'informatique cognitive dans la psychothérapie : Opportunités et risques » mérite un résumé complet. Dirk Helbling est professeur de Computational Social Science à l'ETH de Zurich.

Le sujet doit nous, les thérapeutes, nous occuper réellement et nous faire réfléchir, nous ne pouvons pas simplement ignorer que l'informatique cognitive va être utilisée ou est déjà utilisée aussi dans la psychothérapie, comme dans d'autres domaines du secteur de la santé.

Les quantités de données basées sur l'informatique permettent de nouvelles opportunités d'optimisation de l'efficacité de la médecine, mais elles cachent aussi des risques. Les motsclés sont : Médecine personnalisée, établissement optimisé de diagnostic et assistance thérapeutique sur la base de lignes directrices. Et bien sûr, l'intérêt des organismes de financement dans l'utilisation de ces données est la baisse des frais de santé ou la sélection des risques des assureurs.
Le World Wide Web (WWW) est un réseau mondial d'informations (inventé à l'origine à des fins de renseignements militaires) et constitue un marché mondial de données. Les réseaux sociaux sont des nœuds dans le WWW dans lesquels des données sont générées et transmises. La quantité de données est tellement immense qu'il est impossible que des personnes seules puissent les analyser. Lintelligence artificielle est utilisée pour l'analyse. Dans le monde entier, 700.000 interrogations Google et 500.000 posts Facebook ont lieu en une minute. Nos mouvements génèrent des traces de données qui peuvent même être vues en temps réel.

Le paradigme des mégadonnées est : Si nous avions seulement assez de données, nous n'aurions alors plus besoin de théories, les données révèleraient la vérité. Depuis lors, autant de données que possible sont collectées afin de comprendre le cerveau et la psyché. C'est un rêve et en même temps un cauchemar!

Internet est déchiffré : On enregistre combien de temps vous restez où lorsque vous surfez, ce que 